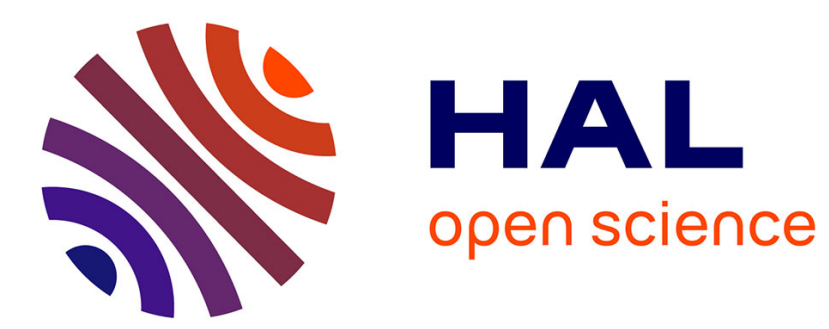

\title{
Les inscriptions
}

Jacques Gascou

\section{To cite this version:}

Jacques Gascou. Les inscriptions. Gallia - Fouilles et monuments archéologiques en France métropolitaine, 1990, Le mausolée de Cucuron (Vaucluse), 47, pp.194-201. 10.3406/galia.1990.3161 . hal02477687

\section{HAL Id: hal-02477687 \\ https://hal.science/hal-02477687}

Submitted on 13 Feb 2020

HAL is a multi-disciplinary open access archive for the deposit and dissemination of scientific research documents, whether they are published or not. The documents may come from teaching and research institutions in France or abroad, or from public or private research centers.
L'archive ouverte pluridisciplinaire HAL, est destinée au dépôt et à la diffusion de documents scientifiques de niveau recherche, publiés ou non, émanant des établissements d'enseignement et de recherche français ou étrangers, des laboratoires publics ou privés.

\section{(이) $\$$}

Distributed under a Creative Commons Attribution - NonCommercial - NoDerivatives| 4.0 
l'iris creux, absent de Cucuron, l'est également des sculptures de l'Italie du Nord, notamment dans la région alpine. En particulier, il ne figure pas sur l'arc de Suze. Par conséquent, nous serions porté à voir dans les masques de Cucuron la main d'un artiste, sinon d'origine ilalique, du moins formé à la manière de l'Italie du début de l'Empire, alors que dans le cas des exemples de Vienne ou de Vaison-la-Romaine, on a l'impression d'un style plus "gallo-romain" et sans doute déjà du $\mathrm{II}^{\mathrm{e}} \mathrm{s}$.

\section{Henri I,avagne.}

\section{LES INSCRIPTIONS}

\section{INSCRIPTIONS DÉCOUVERTES AU VOISINAGE DU MAUSOLÉE}

1 Trouvée en avril ou mai 1970. Conservée au musée de Cucuron.

Fig. 47. Plaque de calcaire rectangulaire brisée obliquement dans sa partie inférieure. Hauteur: $35,5 \mathrm{~cm}$; largeur : $50 \mathrm{~cm}$; épaisseur (incomplète) : $15,5 \mathrm{~cm}$. Hauteur des lettres : lignes 1 et $2: 5,5 \mathrm{~cm}$; ligne $3: 4,4$ à $5,2 \mathrm{~cm}$; ligne $4:$ incomplète.

Inédite. Révisée en novembre 1986.

$$
\begin{aligned}
& \text { [. ?] VCVTTECTO } \\
& \text { [. ?]COMMIVS } \\
& \text { [..]ṬVRNINVS } \\
& {[-.--.--] M}
\end{aligned}
$$

Au début de la 1. 1, la surface, endommagée, pouvait contenir une lettre avant le premier $\mathrm{V}$. Il est même probable qu'un caractère y était inscrit : en effet, à la 1.3 , il y avait certainement deux lettres avant le $\mathrm{T}$ initial partiellement conservé. La seconde de ces lettres se trouvait nécessairement à l'aplomb du premier $\mathrm{V}$ de la l. 1. Or, la mise en page, contrastant avec la qualité relativement médiocre de la gravure, paraît avoir été très soignée, car la dernière lettre des 1.2 et 3 est gravée juste à la verticale du dernier signe de la l. 1. Il est donc présumable que la première lettre de la l. 1 était aussi exactement à l'aplomb de la première de la l. 3 . Il est possible que subsiste avant le $\mathrm{V}$ une très faible trace d'un fragment de haste, mais on ne peut l'affirmer, compte tenu de l'état de la pierre en cet endroit. La lecture [L]ucuttecto est cependant probable, car on ne peut guère envisager un autre premier élément de ce nom que lucu- (=lugu-), cf. infra;

1. 2 : avant le gentilice Commius, il y a place pour un prénom abrégé en une lettre. Bien que le nom du dédicant ait pu ne comporter qu'un gentilice

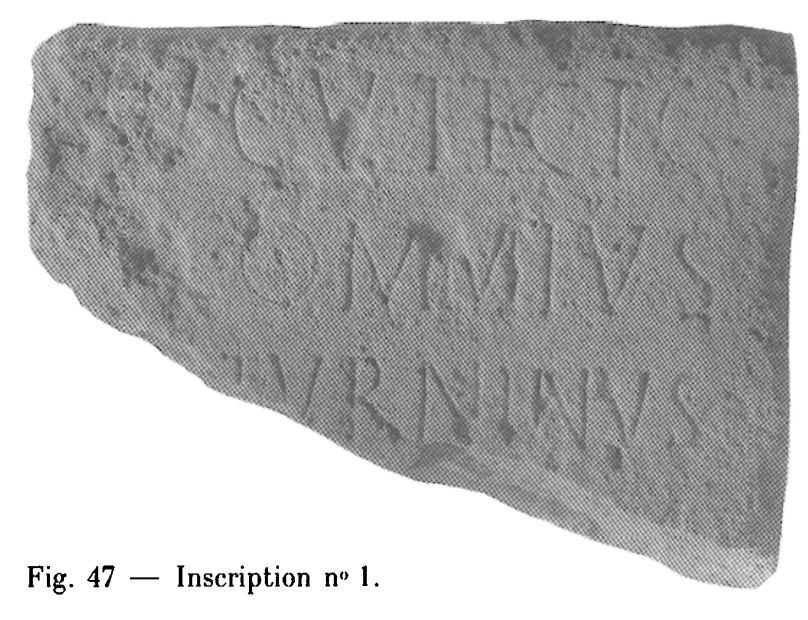

et un surnom, on supposera que le prénom était effectivement gravé pour la même raison de mise en page qu'on vient d'invoquer: l'initiale du prénom devait se trouver juste au-dessus de la première lettre de la l. 3 ;

l. 3 : la restitution [Sa/turninus est évidente;

1. 4 : on distingue à la fin un fragment de barre oblique formant un angle aigu avec le haut d'une haste verticale. Il ne peut s'agir que de la fin d'un $M$.

On peut, à partir de là, restituer la formule votive bien connue: [u(otum) s(oluit) l(ibens] $m$ (erito). Au total, on comprendra ainsi l'ensemble du texte :

[L]ucuttecto / [.] Commius / [Sa]turninus / [u(otum) s(oluit) l(ibens)] m(erito).

"Envers Lucuttectus, [.] Commius Saturninus s'est acquitté de son vœu, de bon gré et à juste titre».

Lucuttectus : nom de divinité gallo-romaine jusqu'à présent inconnu. M. M. Lejeune, que nous 
remercions de ses informations (lettre du 28/1/1987), considère comme vraisemblable la lecture [L]ucuttecto. Mais il estime que l'élément lucu- est ici l'équivalent de lugu-, "avec le banal flottement galloromain $\mathrm{G} / \mathrm{G}$ ". "On aurait alors deux termes de composés : lugu-, -tecto-, avec, à la jointure une (banale aussi) gémination expressive». M. Lejeune se refuse d'autre part à "traduire" ce composé. Sur ces éléments, on lira D. E. Evans, Gaulish Personal Names, Oxford, 1967, p. 219-221 (pour lugu-) et p. 265-266 (pour teclo-) : l'étymologie et la signification de lugu- ont fait l'objet de maintes spéculations qui ne débouchent que sur des conjectures qu'il serait vain d'énumérer. On retrouve cet élément dans le nom de $\operatorname{Lug}(u)$ dunum. Quant à l'élément tecto-, Evans le met en relation avec l'indo-européen *leg-, "couvrir". On le retrouve dans divers noms gaulois : Atectius, Atectorigiana, Atectorix..., ainsi que dans celui du peuple des Tectosages.

Le nom de Lucultectus est à ajouter à celui des nombreuses divinités topiques, portant une dénomination celtique ou celto-ligure, qui sont attestées en Gaule Narbonnaise et qu'a étudiées A. Grenier (Aspects de la religion romaine en Provence, CRAI, 1954 , p. 328-335).

Commius : sur ce gentilice d'origine celtique, qui correspond au gaulois Commios, cf. A. Holder, AltCeltischer Sprachschatz, I, Leipzig, 1896-1907, col. 1074-1078, s.v. Commios, Commiios, et W. Schulze, Zur Geschichte Laleinischer Eigennamen, Berlin, 1904, p. 426. Cf. aussi le Thesaurus Linguae Latinae, Onomasticon, II, col. 547. Il n'était pas jusqu'à présent connu en Gaule Narbonnaise : il n'apparaît pas dans l'index du CIL XII, ni dans celui des ILGN d'Espérandieu. Le nom Commius est celui d'un chef atrébate mentionné à de très nombreuses reprises dans le Bellum Gallicum: cf. César, B.G., 4, 21, 7 ; 27, 2 ; 35, 1 ; 5, 22, 3; 6, 6, 4; 7, 75,$5 ; 76,1 ; 76,3$, etc. On trouve Commius et Commia comme gentilice en CIL V, 425 (Zepich), comme gentilice ou nom unique en CIL XIII, 3313 (Reims) et comme nom unique sur un vase de Reims (CIL XIII, 10010,618).

Saturninus : sur ce surnom, cf. I. Kajanto, The Latin Cognomina, Helsinki, 1965, p. 213 et G. Alföldy, Die Personennamen in der römischen Provinz Dalmatia, Heidelberg, 1969, p. 288. C'est un surnom très fortement répandu dans toutes les parties de l'Empire, mais surtout en Afrique (1 163 exemples sur un total de 2507 dans l'ensemble des volumes du $C I L)$ et dans les provinces celtiques. On en relève au $C I L$ XII, 43 exemples au masculin (et un exemple douteux dans les ILGN) et 22 au féminin.
On a au total affaire dans cette inscription à une onomastique à dominante celtique : c'est évident si l'on considère le nom du dieu et le gentilice du dédicant. Quant au surnom, s'il n'a rien de celtique, il est néanmoins très bien attesté en pays celtique.

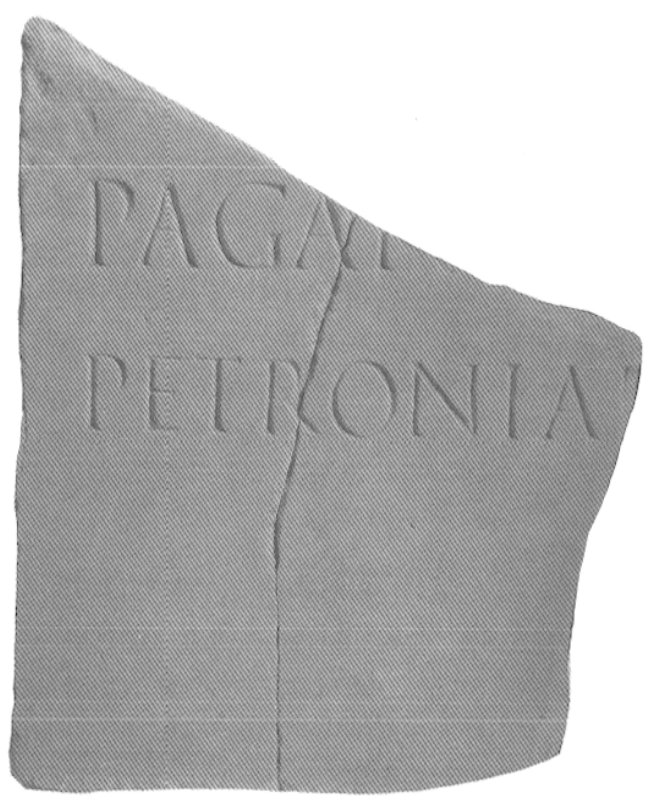

Fig. $48-$ Inscription no 2 .

2 Trouvée à la même époque que l'inscription précédente. Conservée au musée de Cucuron.

Fig. 48. Plaque de marbre blanc en deux morceaux jointifs, brisée au sommet, à droite et au bas, mais le texte est complet au bas, car un très grand "blanc" existe entre la deuxième ligne conservée et la partie inférieure conservée de la plaque. On aperçoit une trace de moulure au bas. Hauteur : $45 \mathrm{~cm}$; largeur : $40 \mathrm{~cm}$; épaisseur: $3 \mathrm{~cm}$. Hauteur des lettres : ligne $1: 5 \mathrm{~cm}$; ligne $2: 4,5 \mathrm{~cm}$.

Inédite. Révisée en novembre 1986.

$$
\begin{gathered}
\text { PAGAN [--- ] } \\
\text { PETRONIAẸ[---] } \\
\text { Pagan [i-- - ] / Petroniae[---] }
\end{gathered}
$$

Très belle gravure pouvant appartenir au $\mathrm{I}^{\mathrm{er}}$ ou au $\mathrm{II}^{\mathrm{e}} \mathrm{s}$. On ne peut déterminer l'étendue de la lacune à droite. Il est cependant vraisemblable qu'on avait au moins, après le mot pagan [ $i$ ], un adjectif à valeur toponymique et, après le gentilice Petroniae, un surnom. La restitution Pagan[i] / Petronia[ni] à laquelle nous avons un moment pensé et qui nous eût révélé le nom d'un nouveau pagus d'Aquae Sextiae nous paraît impossible, car après Petronia on aperçoit une haste dont la partie supérieure ne comporte 
pas le petit appendice très fin partant vers la gauche que l'on distingue au sommet du début du $\mathrm{N}$ conservé à la ligne 2 . En revanche, le sommet de cette haste est tout à fait semblable à celui de la haste du $\mathrm{E}$ gravé à la même ligne. De surcroît, il semble bien que l'on distingue le départ d'un trait formant un angle droit avec la haste et qui appartient sans doute à la barre horizontale supérieure d'un E. Pour ces raisons, la lecture Petroniae nous paraît assurée.

Cette inscription est vraisemblablement une dédicace honorifique adressée à une femme par les membres d'un pagus appartenant au territoire d'Aquae Sextiae. On peut la comparer avec plusieurs inscriptions citées ci-après dans lesquelles un pagus ou les membres d'un pagus font une dédicace à un personnage (nous laissons de côté les dédicaces impériales).

- $A E, 1900,96=I L S, 6596$ :

Sex(to) Mecio, Sex(ti) f(ilio), Sab(atina tribu), । Marcello, II(uiro) q(in)q(uennali) Salur/ni(ae), $q$ (uaestori) kal(endarii) r(ei) p(ublicae) et aliment(orum), / curat(ori) pagi Lucreti, / iuueni o(ptimo) et amant(issimo), / ob multa eius bene/ficia pagani pagi / s(upra) s(cripti) in se conlata / dignissimo po/suerunt. / L(ocus) d(atus) ex d(ecreto) d(ecurionum).

Pour la compréhension de ce texte, il faut intervertir la place des groupes de mots pagani pagi s.s. et in se conlata.

- $C I L$ IX, $3306=I L S, 932^{\mathrm{a}}$ :

Q(uinto) Vario, Q(uinti) f(ilio), / Ser(gia tribu), Gemino, q(uaestori), / pagus Vecellanus.

- $C I L$ IX, $3311=I L S, 6532$ :

Q(uinto) Octauio, L(ucii) f(ilio), / Sagittae, quinq(uennali) II, pagus Boedinus.

- $\quad C I L$ XII, $594=I L S, 6988$ :

[PJagani pagi Lucreti, qui sunt fini/bus Arelatensium loco Gargario, Q(uinto) Cor(nelio), I Marcelli lib(erto), Zosimo, IIIIIIuir(o) Aug(ustali) col(onia) Iul(ia) / Paterna Arelate ob honorem eius ...

Suit un long développement où sont exprimés les motifs de la reconnaissance des pagani.

- $C I L$ XII, $1114=I L S, 6989$ :

C(aio) Allio, C(aii) fil(io), / Volt(inia tribu), Celeri, / IIIIuir(o), flam(ini), / augur(i) col(onia) I(ulia) | Apt(a), ex V dec(uriis), / Vordenses pa/ga/ni | paltro/no.

Les raisons pour lesquelles Petronia [---] a été honorée sont naturellement impossibles à déterminer. On ne peut guère penser à un patronat, comme en CIL XII, 1114, car les femmes ayant exercé un patronat sur une ville, un uicus ou un pagus, toutes de famille clarissime, sont extrêmement rares : L. Harmand ne dénombre que cinq femmes patronnes sur un total de 670 patrons de "collectivités publiques ${ }^{1}$. Petronia /---/ était-elle la fille ou la femme d'un personnage important qui aurait pu rendre des services au pagus aixois qui se trouvait à Cucuron? On connaît un Lucius Petronius Sabinus qui exerça la procuratèle du patrimoine de la province de Gaule Narbonnaise sous le règne conjoint de Marc Aurèle et de Commode (176-180) ${ }^{2}$. Sa fille, Petronia Sabina, est honorée sur une inscription d'Ancône, dont il était le patron et où il avait dû se retirer après avoir achevé sa carrière ${ }^{3}$. Peut-on penser que L. Petronius Sabinus aurait pu rendre quelque service au pagus de nom inconnu que nous révèle l'inscription de Cucuron, et que, pour cette raison, les pagani auraient honoré sa fille? Il ne s'agit là que d'une conjecture très peu sûre, à laquelle on peut d'ailleurs opposer une objection : l'inscription ne comporte que deux lignes ${ }^{4}$ et, à la première d'entre elles, il ne peut guère y avoir eu autre chose après pagan[i] qu'une épithète toponymique se rapportant directement à ce substantif, ou encore le mot pagi suivi de cette même épithète ${ }^{5}$. Or, à la deuxième ligne, si la femme, objet de la dédicace, était la fille du procurateur du patrimoine de Gaule Narbonnaise, on aurait dû avoir, non seulement le nom Petroniae [Sabinae], mais encore, comme dans l'inscription d'Ancone, l'indication de sa relation de parenté avec ce haut personnage et, à tout le moins, son titre de procurateur, sinon même l'ensemble de son cursus. Or, cela ferait un texte

1 I. Harmand, Le palronal sur les collectivilés publiques des origines au Bas-Empire, Paris, 1957, p. 281-282. Il est à noter que L. Harmand a oublié le patron de pagus de CIL XII, 1114.

2 CIL IX, 5898 (= ILS, 1386), cf. II.-G. Pri.Aum, Les Fastes de la province de Narbonnaise, 30' suppl. à Gallia, Paris, Éd. du GNRS, 1978, p. 124-125.

3 Inscription citée à la note précédente, dont voici le texte : Petroniae / Sabinae,/ filiae / L. Petroni Sabini / p(rimi) p(ili) bis, / procurator(is) Aug(ustorum duorum) / rationis hereditatium, item prouinciae / Narbonensis,| patron(i) coloniae,/ decuriones.

4 L'espace entre les deux lignes conservées est de $5 \mathrm{~cm}$. L'espace est très légèrement supérieur entre le $P$ initial de la ligne 1 et la cassure du haut de la pierre. Mais comme cette cassure descend en biais, on pourrait concevoir qu'il y ait eu une ligne inscrite avant la première ligne conservée : elle aurait pu commencer en retrait par rapport au début de cette dernière. Mais on voit mal, dans une dédicace de ce type, ce qui aurait pu précéder. On doit plutôt penser que, comme en CII, XII, 594, l'inscription commençait par la mention des pagani auteurs de la dédicace.

5 De la même façon qu'en $C I I$, XII, 594 : /P/agani pagi Lucreti. 
beaucoup trop long pour qu'il ait pu être gravé sur la seule deuxième ligne. Il est donc préférable de penser que la Petronia [--] honorée par les pagani de Cucuron était la femme ou la fille d'un notable local, ou encore une riche propriétaire ou une prêtresse de la cité d' $\Lambda \mathrm{ix}^{6}$, qui aurait pu rendre des services au pagus ou y occuper une importante position. Le gentilice Petronius, qui est partout largement répandu (cf. (. Alföldy, op. cit., p. 108), n'était cependant pas jusqu'à présent connu dans la cité antique d'Aix.

Que le pagus révélé par cette inscription ait fait partie du territoire d'Aquae Sextiae ne saurait faire de doute depuis la démonstration du chanoine J.-H. Albanès (Gallia Christiana nouissima, I, Aix, Apt, Fréjus, Gap, Riez el Sisteron, Montbéliard, 1899, p. 177-178) : la plus grande partie du pays compris entre la Durance et le Lubéron, et notamment la région où se trouve Cucuron, appartenait dans l'Antiquité à la cité d'Aquae Sextiae et non pas à celle d'Apt comme le voulait $O$. Hirschfeld, CIL $\mathrm{XII}$, p. 138. On connaissait jusqu'à présent un et peut-être deux pagi du territoire d'Aix.

- Un texte épigraphique aujourd'hui perdu, que Peiresc a signalé parmi les inscriptions d'Aix-enProvence, sans toutefois indiquer en quel lieu du territoire aixois il avait été découvert, mentionne le

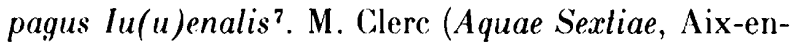
Provence, 1916, p. 204) rapprochait ce nom de celui de la Jouine, «ruisseau formé de deux autres, la Grande et la Petite Jouine, venus l'un de Bouc, l'autre de Cabriès, et qui se jette dans l'Arc, en face de Saint-Pons, à une dizaine de kilomètres audessous d'Aix" (ibid., p. 205, cf. p. 207). Comme la Jouine formait, selon M. Clerc, la limite entre les territoires d'Aix et d'Arles, ce pagus aurait compris la partie occidentale de la cité d'Aix. En réalité, l'étymologie qui rattache le nom de la Jouïne à Iuuenalis ne repose que sur une vague ressemblance phonétique et apparaît comme une conjecture très peu sùre. On se gardera donc de tenter de situer ce pagus à l'intérieur du territoire aixois, en l'absence de données plus précises.

6 La seule prêtresse de la colonie d'Aquae Sexliae que l'on connaisse est mentionnée dans une inscription disparue, lue autrefois par Solier, Belleforest, Peiresc et Bouche (CIL XII, 519), et attestant l'existence d'une flaminic(a) Auguslae II (l'indication chiffrée II (= ilerum), tout à fait insolite auprès du titre de flamine d'Auguste ou de flaminique d'Augusta, pose un problème).

7 Il s'agit d'une dédicace pour le salut de Néron (CIL XII, 512): Pro salute / Neronis Claud(ii) / Caesaris Aug(usti) / Ger(manici), p(atris) p(atriae) sacr(um),/ /p Jagus Iu(u)enalis. Néron étant pater patriae à la fin de 55 ou au début de 56, l'inscription est datable des années 55-68.
- D'après L.-A. Constans (Arles antique, Paris, 1921 , p. 71 et 73 ), il aurait existé un second pagus dans le sud du territoire aixois, à proximité de la vallée de l'Huveaune: cet auteur se fonde sur l'inscription CIL XII, 594 (cf. supra), trouvée à Saint-Jean de Garguier, à $2 \mathrm{~km}$ au nord-ouest de Gémenos, où se lisent les mots /p Jagani pagi Lucreti, qui sunt finibus Arelatensium loco Gargario. A l'inverse de C. Jullian (Bulletin Epigraphique du Midi de la Gaule, 5, 1885, p. 175-183) qui en infère que tous les pagani pagi Lucreti ressortissaient au locus Gargarius, et donc à la cité d'Arles, L.-A. Constans pense qu'il est question dans cette inscription «d'une partie des pagani qui était sur le territoire d'Arles, par opposition à un autre groupe qui était sur le territoire d'Aix». Dans ce cas, le pagus Lucretius se serait étendu pour une part sur le territoire d'Arles et pour une autre part sur celui d'Aix. Cette interprétation, séduisante sans être certaine, reçoit une certaine confirmation des deux bornes frontières (aujourd'hui perdues) mentionnant, l'une les limites du territoire d'Arles, l'autre celles du territoire d'Aix, qui ont été signalées au xviri s. dans un ouvrage manuscrit du père Bougerel (CIL XII, 531$, d-e $)^{8}$, et qui ont été trouvées à Gémenos : elles prouvent en effet que le territoire aixois commençait à une très faible distance du locus Gargarius.

En revanche, le pagus Matauonicus, mentionné dans une inscription de Cabasse (CIL XII, $312=$ ILN-Fréjus, 164), et qui selon M. Clerc, (op. cit., p. 199, 205, 207) appartenait au territoire d'Aquae Sextiae, doit plutôt être rattaché au territoire de Fréjus ${ }^{9}$.

Pour être complet, il faut enfin mentionner l'opinion de M. Clerc (op. cit., p. 207 et 276-277) qui croyait pouvoir affirmer l'existence d'un autre pagus de la cité d'Aquae Sextiae : il se fondait pour cela sur une inscription trouvée sur la colline du Castellar à Cadenet (CIL XII, 1064), qui est dédiée Dexiuae et Caudellensibus. Comme certains érudits ont estimé que le mot Caudellenses était un ethnique désignant

8 La réalité de ces bornes a èté parfois mise en doute, cf. J.-H. Albanès, Gallia Christiana nouissima, I, p. 56. Mais C. Jullian (Bulletin Égraphique, 5,1885 , p. 15) a montré que la haute vallée de l'Huveaune, au sud du diocèse médiéval d'Aix, au voisinage de laquelle se trouve Gémenos, appartient sans doute dans l'Antiquité, pour une grande part, au territoire d'Aquae Sextiae, comme incite à le penser la présence de la tribu Voltinia, celle d'Aix, à Saint-Zacharie, à quelques kilomètres au nord-est de Gémenos. De ce fait, l'authenticité des bornes signalées par le père Bougerel apparaît comme moins sujette à caution.

9 J. Gascol, M. JanON, ILN-Fréjus, 44 suppl, à Gallia, Paris, Ed. du CNRS, 1985, p. 26-27. 
les habitants du uicus de Cadenet, Clerc pensait que "les Caudellenses étaient les habitants de la région de Cadenet-Pertuis, dont le Castelar a été l'une des principales résidences». Il en déduisait que les Caudellenses devaient former un pagus de la cité d'Aix, qui se serait étendu sur toute la région située entre Durance et Lubéron. Si l'on pouvait suivre Clerc dans son raisonnement, on tiendrait la restitution de la première ligne de l'inscription de Cucuron : Pagan [i Caudellenses] ou Pagan[i pagi Caudellensis]. En réalité, il est impossible de suivre cet auteur : les Caudellenses, dans l'inscription de Cadenet, sont placés sur le même plan que la déesse Dexiua et, comme l'inscription se termine par la formule u.s.l.m., il est évident que l'on a affaire à une inscription votive englobant Dexiua et les Caudellenses. Les Caudellenses sont donc sûrement des divinités collectives et non pas les habitants de la région de Cadenet ${ }^{10}$.

Nous conclurons que l'inscription de Cucuron nous révèle l'existence d'un pagus aixois au nom inconnu qui englobait le territoire de Cucuron mais dont il est impossible de préciser l'extension.

3 Trouvée à la même époque que les deux inscriptions précédentes. Conservée au musée de Cucuron. Fig. 49. Six fragments de marbre blanc, dont deux anépigraphes, appartenant sans doute à la même inscription. Le fragment a, formé de deux morceaux jointifs, porte des moulures dans sa partie supérieure et appartenait donc à la première ligne de l'inscription. Un second fragment (b) est brisé de tous côtés et ne comporte aucune moulure. Deux autres fragments (c et d) ont des moulures dans leur partie inférieure et appartenaient à la dernière ligne. Les deux fragments anépigraphes devaient appartenir, l'un à la partie supérieure, l'autre à la partie droite de la plaque.

Inédite.

Fragment a. Hauteur : $18,5 \mathrm{~cm}$;- largeur : $10 \mathrm{~cm}$; - épaisseur : $5,5 \mathrm{~cm}$. Hauteur de la lettre : $9,2 \mathrm{~cm}$.

$$
\text { -.- }] \mathbf{E}[---
$$

Fragment b. Hauteur : $4,3 \mathrm{~cm}$; - largeur : $8,6 \mathrm{~cm}$; - épaisseur : $3 \mathrm{~cm}$. Hauteur de la lettre : non mesurable.

On distingue un fragment de haste qui pouvait être verticale, horizontale ou oblique, appartenant à une lettre impossible à identifier.

10 Dans le même sens, cf. G. Barruol, Les peuples préromains du sud-est de la Gaule. Élude de géographie historique, I ${ }^{\mathrm{er}}$ suppl. à la $R A N$, Paris, 1969, p. 204, note 3.

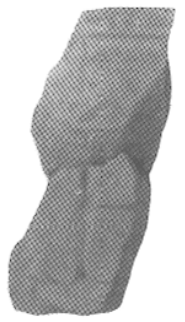

a

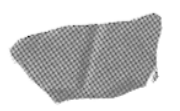

b

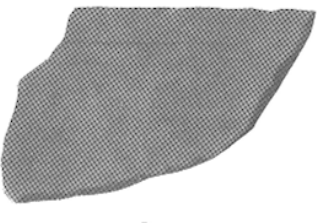

e

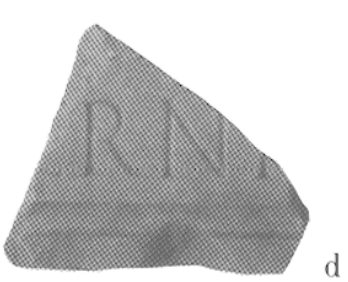

Fig. 49 - Inscription no 3 .

Fragments $\mathrm{c}$ et d. Hauteurs : $14,5 \mathrm{~cm}(\mathrm{c}) ; 15,7 \mathrm{~cm}$ (d); - largeurs : $13,5 \mathrm{~cm} \mathrm{(c);20,5} \mathrm{cm} \mathrm{(d);} \mathrm{-}$ épaisseur : $4,5 \mathrm{~cm}(\mathrm{c}$ et $\mathrm{d})$. Hauteur des lettres : $4,7 \mathrm{~cm}(\mathrm{c}$ et $\mathrm{d})$.

$$
\begin{gathered}
c \quad d \\
\text { - - - MA [.]ẸRI[--- }]
\end{gathered}
$$

Il est probable qu'il n'existait qu'un faible espace entre les deux fragments et que l'on peut restituer : ---] Ma[t]erni [---]

L'inscription est trop fragmentaire pour que l'on puisse préciser sa nature exacte. On peut seulement affirmer qu'elle comportait, au génitif, le nom unique ou le surnom Maternus. Ce cognomen est très fréquent en Narbonnaise : on recense 42 exemples de Maternus au CIL XII, deux dans les ILGN et 31 exemples de Materna au CIL XII (et aussi deux attestations dans les $I L G N)$. Il est en général surtout connu dans les provinces celtiques (cf. à ce sujet I. Kajanto, op. cit., p. 303) : sur un total de 285 exemples connus de Maternus ou Materna dans l'ensemble des volumes du $C I L, 187$ se trouvent dans les trois volumes II, XII et XIII, c'est-à-dire dans des zones celtiques (Espagne et Gaules). On ne connaissait jusqu'à présent dans le territoire d'Aixen-Provence qu'un seul exemple de ce nom, attesté au féminin, à Saint-Canadet, dans la commune du Puy-Sainte-Réparade (ILGN, 84). On connait désormais, outre celui qui se rencontre ici, un autre Maternus mentionné dans l'inscription $n^{0} 6$ (cf. ciaprès). 


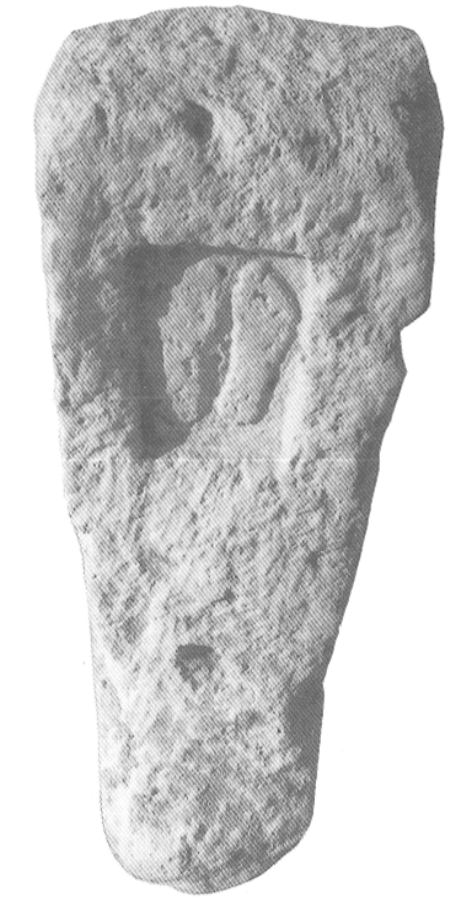

Fig. 50 - Stèle anépigraphe no 4 .

\section{STĖLE ANÉPIGRAPHE}

4 Bien qu'il ne s'agisse pas d'une inscription, il nous a paru utile de joindre aux documents qui précèdent une stèle trouvée également au voisinage du mausolée de Cucuron à la même époque.

Fig. 50. Il s'agit d'une pierre de forme vaguement trapézoïdale, portant, dans un cartouche rectangulaire en creux, deux pieds sculptés. Comment interpréter ce monument?

La coutume de représenter des empreintes de pieds, répandue en Orient comme dans le monde gréco-romain, dans l'univers païen comme dans l'univers chrétien, a donné lieu à une abondante littérature. Nous nous bornerons à renvoyer à quelques travaux qui eux-mêmes contiennent de nombreuses références bibliographiques : M. Guarducci, Le impronte del "Quo Vadis» e monumenti affini, figurati e epigrafici, Rendiconti della Pontificia Accademia Romana di Archeologia, 19, 1943, p. 305-344; - L. Castiglione, Vestigia, Acta Archaeologica Academiae Scientiarum Hungaricae, 22, 1970, p. 95-132; Id., Footprints of the Gods in India and in the Hellenistic World; Influence or Parallelism? Annales Archéologiques de Syrie, 21, 1971, p. 25-36.

Nous n'énumérerons pas les sens multiples que, selon les temps et les lieux, cette symbolique a pu revêtir; nous rapprocherons seulement ce document de deux pierres contenant chacune une inscription gallo-grecque et l'image de deux pieds gravés, rééditées récemment dans $M$. Lejeune, $R I G, \mathrm{I}$, Textes gallo-grecs, 45 ${ }^{\mathrm{e}}$ suppl. à Gallia, Paris, 1985.

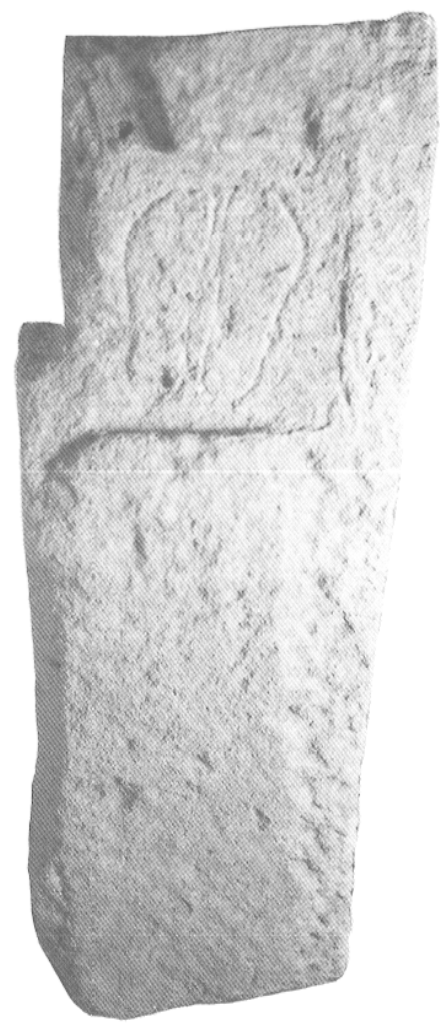

Fig. 51 - Stèle anépigraphe de Cadenet.

- Une stèle trouvée sur l'oppidum du Castellar, à Cadenet $(R I G, \mathrm{I}, \mathrm{G}-112$, p. 138-140), et qui paraît avoir disparu depuis la fin du $\mathrm{XIX}^{\mathrm{e}} \mathrm{s}$. : le texte de l'inscription est d'interprétation difficile, mais M. Lejeune estime qu'il s'agit d'une épitaphe. D'après un dessin que Ch.-A. Roland (Cadenet historique, I, Paris, 1837, p. 261) a donné de ce monument, on sait qu'au-dessous de l'inscription étaient gravées deux empreintes de pieds, la pointe dirigée vers le haut, comme sur la stèle de Cadenet.

- Un cippe de Saint-Saturnin d'Apt conservé au musée Calvet d'Avignon (RIG, I, G-152, p. 201204) : il comporte des noms propres dont la disposition correspond à la structure : "Un Tel à Une Telle». Il s'agit soit d'une inscription qu'un fidèle adresse à une déesse, soit plutôt, comme incline à le penser M. Lejeune, d'une épitaphe qu'un dédicant adresse à une défunte. Au-dessous de l'inscription sont gravées les empreintes parallèles de deux pieds dont la pointe est dirigée vers le bas.

De ces deux documents, trouvés le premier à $7 \mathrm{~km}$, le second à $20 \mathrm{~km}$ de Cucuron, on peut conclure avec quelque probabilité que la stèle anépigraphe de Cucuron est elle aussi une pierre à destination funéraire.

On peut enfin établir un parallèle entre le document qui nous occupe et une autre stèle anépigraphe trouvée récemment sur l'oppidum du Castellar de Cadenet et qui est conservée au musée 
de Cucuron (a priori, rien ne permet de supposer qu'il s'agisse de la partie inférieure de la stèle inscrite de Cadenet signalée ci-dessus) : elle aussi comporte l'image de deux empreintes de pieds gravées (fig. 51), dont la représentation est tout à fait comparable à celle de la stèle de Cucuron. Il est permis de supposer qu'elle avait une destination semblable.

\section{INSCRIPTIONS TROUVÉES RÉCEMMENT Aं CUCURON}

5 Découverte en 1950 "en labourant un champ, à $200 \mathrm{~m}$ au sud-ouest de la ferme de l'Aumane, à michemin entre Cucuron et Cabrières. Aux alentours, débris d'amphores, de legulae et de tessons communs»(H. Rolland). Signalée d'abord en 1950 de façon très imprécise par J. Sautel et sans indication du texte" ${ }^{1}$, elle le fut à nouveau (avec le texte), d'après G. Barruol et P. Martel, par H. Rolland, suns référence à l'indication de Sautel. L'inscription est conservée à Cucuron, au château de Gabaru, chez M. le général Bombes de Villiers.

Fig. 52. Autel de calcaire avec base, couronnement et focus au sommet. Puluini de part et d'autre du focus. Le soc de la charrue a endommagé la surface, surtout au-dessous de la première ligne. Réglures très nettes. Hauteur : $58 \mathrm{~cm}$; - base, largeur : $33 \mathrm{~cm}$; épaisseur : $26 \mathrm{~cm}$; - couronnement, largeur : $34 \mathrm{~cm}$; épaisseur : $27 \mathrm{~cm}$; - dé, hauteur : $34 \mathrm{~cm}$; largeur : $26 \mathrm{~cm}$; épaisseur : $21 \mathrm{~cm}$. Hauteur des lettres : 4 à $4,5 \mathrm{~cm}$.

J. Sautel, Gallia, 8, 1950, p. 138; H. Rolkand, ibid., 22, 1964, p. 560 (sans photographie ni fac-similé) $=A E, 1965,190$. Révisée en août 1987.

\section{$\mathrm{GE} \cdot \mathrm{NI} \cdot \mathrm{O} \cdot \mathrm{AD}$ $\mathrm{CO} \cdot \mathrm{RO} \cdot$}

Points triangulaires disposés après chaque syllabe, sauf à la fin de la première ligne. "A la deuxième ligne, peut-être RIO" (H. Rolland). Cette lecture serait en contradiction avec les deux autres dédicaces à la même divinité (cf. infra). De surcroît, il n'y a pas place pour un $I$ entre le $R$ et le $O$ final partiellement conservé. C'est une des rainures creusées à la surface de l'inscription par le soc de la charrue qui a pu faire croire à la présence d'un I.

\section{Genio Ad/coro.}

"Au génie Adcorus».

Le dieu (nommé deux fois "génie») Adcorus n'est attesté que dans le territoire antique d'Aix. On le

11 Cf. infra, bibliographie. J. Sautel présente ainsi cette inscription : "Un autel, au Génie (sic), bien conservé, avec récipient pour les libations, a été découvert à Cucuron, dans la propriété de Laumane». Les mesures indiquées par cet auteur sont fausses.

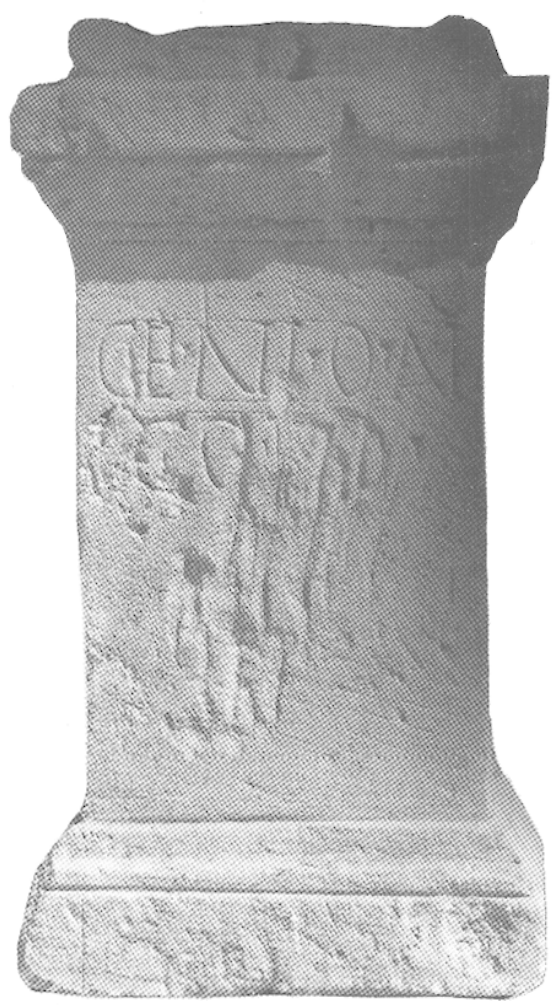

Fig. $52-$ Inscription $n^{\circ} 5$.

trouve, avec deux graphies différentes, Accorus et Acorus, dans deux inscriptions conservées au musée Granet d'Aix-en-Provence : l'une a été découverte à Rognes, au lieu-dit Tournefort, à $4 \mathrm{~km}$ au sud-est de cette localité (CIL XII, 5783) ${ }^{12}$; l'autre, en 1881, à Lançon, au quartier de Lalisson. Cette dernière fut signalée en 1882 par G. Lafaye (Bulletin Epigraphique de la Gaule, 2, p. 126-127) avant d'entrer au CIL XIl sous le numéro $5798^{13}$. Elle fut publiée à nouveau par Y. Burnand, Domitii Aquenses. Une famille de chevaliers romains de la région d'Aix-enProvence. Mausolée et domaine, $5^{e}$ suppl. à la RAN, Paris, 1975, p. 199 , note 389 et photographie pl. VIII, 2 ( $=A E, 1977,533)$. Cet auteur la croyait inédite et la donnait par erreur comme faisant partie du matériel découvert au Grand Saint-Paul, lieu-dit de la commune de Rognes, à environ $2 \mathrm{~km}$ au sudouest de ce bourg.

Adcorus est peut-être un dieu topique, dont la zone d'influence paraît avoir été circonscrite à la partie occidentale du territoire antique d'Aquae

$12 A c / c o / r o$. Avant d'entrer au CIL XII, elle avait été publiée par G. Lafaye (Bulletin Epigraphique de la Gaule, 1, 1881 , p. 224) qui indiquait qu'elle avait èté trouvée une trentaine d'années auparavant.

13 Genio / Acoro. On notera que dans les trois inscriptions mentionnant cette divinité, seul son nom est indiqué, sans précision du nom du ou des dédicants. 
Sextiae ${ }^{14}$. Il s'agit en tout cas d'un dieu protecteur, comme l'indique le mot Genius qui lui est accolé dans deux inscriptions différentes.

Sur Accorus/Acorus, cf. A. Holder, Alt-Celtischer Sprachschatz, op. cit., I, col. 16 et 32 (la forme Adcorus était alors inconnue). Acorus se rencontre aussi comme nom de personne, d'origine sans doute celtique. C'est un nom d'esclave à Sorrente [CIL X, 691 : Acorus, Aug(usti) uer(na)]. Il est utilisé en guise de gentilice dans une inscription de Rome (CIL VI, 10546) : M. Acorus Sulor.

6 Trouvée à Cucuron, au lieu-dit Chabronne, en bordure du val de Peigus, dans la propriété Garcin (section C, dite de Martialis, du cadastre, $2^{\mathrm{e}}$ feuille, n' 236), le 21 septembre 1970. Conservée au musée de Cucuron.

Fig. 53. Autel de calcaire avec base et couronnement, brisé au sommet à gauche et en bas à gauche, ainsi que dans la partie postérieure au sommet et en bas. Au sommet, un puluinus est conservé à droite. Une pelleteuse a endommagé la surface au bas de la ligne 2 et à la ligne 3 . Hauteur : $66 \mathrm{~cm} ;-$ base, largeur : $32 \mathrm{~cm}$; épaisseur maximale : $19 \mathrm{~cm}$; couronnement, largeur maximale : $29 \mathrm{~cm}$; épaisseur maximale : $24,5 \mathrm{~cm}$; - dé, hauteur : $32 \mathrm{~cm}$; largeur : $24 \mathrm{~cm}$; épaisseur : $20 \mathrm{~cm}$. Hauteur des lettres : $5 \mathrm{~cm}$.

Inédite. Révisée en novembre 1986.

$$
\begin{gathered}
\text { SILVANO } \\
\text { T.CORN } \\
\text { MATTERNVS }
\end{gathered}
$$

1. 2 : point triangulaire;

1. 3 : les lettres situées entre le $M$ initial et le groupe des lettres RNVS ne sont pas très aisées à reconnaitre, la surface de la pierre ayant été creusée obliquement en cet endroit par une pelleteuse. On distingue néanmoins le sommet du A et une partie du bas du jambage de droite, une faible partie du sommet et du bas du $\mathrm{T}$ et la partie inférieure du $\mathrm{E}$. Ces trois lettres sont très serreees et occupent un espace un peu moins large que les trois lettres RNV qui leur font suite, elles aussi pourtant assez resserrées. Il ne semble néanmoins pas qu'il y ait eu une ligature TE, comme nous l'avions d'abord cru.

\section{Siluano / T(itus) Corn(elius) / Maternus.} "A Silvain, Titus Cornelius Maternus".

Sur Silvain en Gaule Narbonnaise, cf.

14 A la différence de Ilirschfeld, qui plaçait Lançon dans le territoire antique d'Arles, nous considérons cette ville, qui était au Moyen Age une des paroisses du diocèse d'Aix formant la frontière avec le diocèse d'Arles (cf. J.-II. Al.BanÈs, op. cil., p. 9-12), comme appartenant au territoire d'Aquae Sextiae.

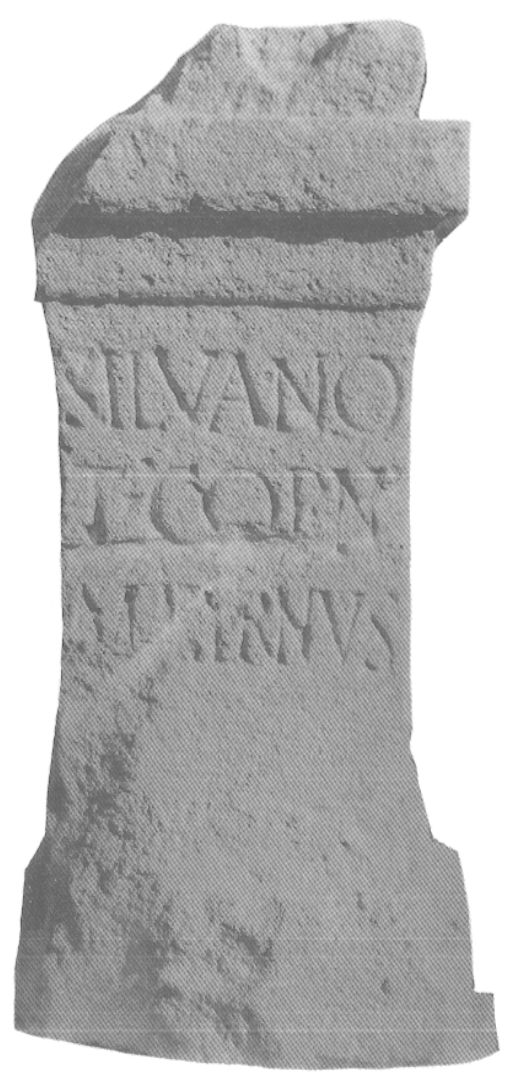

Fig. $53-$ Inscription no 6 .

H. Lavagne, Les dieux de la Gaule Narbonnaise; "romanité. et romanisation, Journal des Savants, juill.-sept. 1979, p. 179-183. Cette divinité de rang inférieur dans le panthéon romain occupe la troisième place en Narbonnaise, si l'on évalue son importance au nombre d'inscriptions qui la mentionnent. Son aire de diffusion principale en Gaule est la vallée du Rhône et de la Saône, le bassin de la Durance, le Comtat-Venaissin, le Viennois. Silvain recouvre très fréquemment le dieu gaulois Sucellus, le "dieu au maillet". C'est le dieu agreste semblable au Silvain italien, défenseur des troupeaux, peut-être aussi le dieu des bûcherons et des conducteurs de radeaux, les ratiarii et, comme Sucellus, le dieu du monde infernal, il est parfois peut-être assimilé à Dispater.

Sur le surnom du dédicant, Maternus, cf. supra, le $n^{0} 3$.

\section{Jacques Gascou*}

* Nous remercions $\mathrm{M}^{\text {me }}$ Marie-Laure Gamerre qui nous a aidé pour la lecture de la dédicace à Adcorus ( ${ }^{\circ} 5$ ) et M. G. Réveillac, photographe au Centre Camille Jullian qui est l'auteur des clichés des inscriptions nos $1,2,5$ et 6 . Nous tenons à exprimer notre gratitude à $\mathrm{M}$. le général Bombes de Villiers, propriétaire du château de Gabaru, qui nous a aimablement autorisé à examiner la stèle qu'il possède et à en faire prendre des clichés ( $\left.n^{\circ} 5\right)$. Nous indiquons plus haut (commentaire de l'inscription $n^{\circ} 1$ ) notre dette envers M.M. Lejeune dans l'interprétation d'une des pierres de Cucuron. 